\title{
A Feasibility Study in Using Facial Expressions Analysis to Evaluate Player Experiences
}

\author{
Chek Tien Tan \\ chektien.tan@uts.edu.au \\ Daniel Rosser
daniel.j.rosser@student.uts.edu.au \\ Sander Bakkes \\ Yusuf Pisan \\ sanderbakkes@gmail.com yusuf.pisan@uts.edu.au
}

Games Studio, Faculty of Engineering and IT, University of Technology, Sydney

\begin{abstract}
Current quantitative methods of measuring player experience in games are mostly intrusive to play and less suited to natural, non-laboratory play environments. This paper presents an initial study to validate the feasibility of using facial expressions analysis for evaluating player experiences. It builds on a prior position that video-based computer vision techniques can provide a less intrusive and more versatile solution for automatic evaluation of game user experiences. A user study was performed on an initial group of participants in a first-person puzzle shooter game (Portal 2) and a social drawing trivia game (Draw My Thing), and the results are shown to support our position.
\end{abstract}

\section{Categories and Subject Descriptors}

H.5.2 [Information Interfaces and Presentation]: User Interfaces: Evaluation/methodology; I.2.1 [Applications and Expert Systems]: Games.

\section{General Terms}

Game User Experience, Facial Expression Analysis, Playtesting

\section{INTRODUCTION}

The evaluation of player experiences is central to the design of digital games. On the whole, the primary goal of digital games is to provide players with appropriate experiences like enjoyment and flow [25]. Within each game, different instances of gameplay also aim to provide short-term experiences like fear, anger and surprise. It is therefore essential in game design to be able to measure whether these experiences are achieved, and perhaps to even measure the extent of each experience in real-time. Research into methods to enable efficient and effective player experience analysis is hence a key area in the digital games domain.

Permission to make digital or hard copies of part or all of this work for personal or classroom use is granted without fee provided that copies are not made or distributed for profit or commercial advantage and that copies bear this notice and the full citation on the first page. Copyrights for components of this work owned by others than ACM must be honored. Abstracting with credit is permitted. To copy otherwise, to republish, to post on servers or to redistribute to lists, requires prior specific permission and/or a fee.

IE '12, July 21 - 22 2012, Auckland, NZ, New Zealand.

Copyright 2012 ACM 978-1-4503-1410-7/12/07...\$15.00.
Research into player experiences is also an area of growing importance in in non-game applications. Recent times have seen the uprising of software gamification, or the concept of using game design methodologies to enhance non-game applications. Examples of gamification adoption can be seen across diverse industries and organizations, from social networking portals [5] to government software [22]. Other than the fundamental usability requirements, many applications are also increasingly having the responsibility of providing gameplay experiences. Player experience research is hence also important to the development of many different types of modern softwares, in order to assess and improve the quality of gameplay experience they provide.

Motivated by the need to capture data in a way that is more efficient, versatile, and does not affect natural gameplay, we build on prior work [27] that proposes a novel computer vision-based technique to infer player experiences automatically based on facial expressions analysis. Facial expressions analysis [12] is the use of automatically recognized facial expressions to infer affective states. It is a video-based approach which is non-obtrusive compared to current physiological approaches. This allows for a more authentic play experience and enables data collection in non-laboratory settings. In addition, current technological advancements favors this approach. The advent of motion detection game consoles like Microsoft's Kinect ${ }^{1}$ and Nintendo's 3DS ${ }^{2}$, video feeds are naturally incorporated into gameplay. For other games, webcams are also relatively cheap and prevalent in most mobile computing devices nowadays, especially when compared to specialized physiological equipment. Facial expressions analysis can be also viewed as a type of psychophysiological approach, which seemed to be underexplored in current player experience research. To the best of our knowledge, no work has been performed to evaluate the feasibility of facial expressions analysis as a basis to infer gameplay experience metrics such as flow, immersion and presence [21].

As further motivation, research in non-game domains have shown good results for inferring other kinds of user experience metrics from facial expressions [29, 12]. Prior psychophysiological approaches [19] have also shown promising

\footnotetext{
${ }^{1}$ http://www.xbox.com/en-US/kinect/

${ }^{2}$ http://www.nintendo.com/3ds
} 
correlations between the measured physiological data and self-reported flow experience. However, before we even venture into inferring these experiences, the question of whether games elicit enough facial expressions, and further more, whether these expressions can be captured robustly, needs to be answered.

As a pilot investigation, this paper provides an initial feasibility study on a group of players in two diverse commercial games (as described in Section 4.1). The aim is to provide some insights into the feasibility of advancing this research on player experience evaluation using facial expressions analysis. Specifically, this paper addresses these questions:

1. whether sufficient facial expressions are elicited during gameplay, and

2. whether these expressions can be captured robustly using a state-of-the-art facial expression recognition system.

In the remainder of this paper, the next section (2) presents related work to justify our motivations on pursuing this research. The section that follows (3) describes our proposed overall methodology. The succeeding section (4) then describes the evaluation we have performed on an initial group of users. The last section (5) concludes with our plan for future work.

\section{RELATED WORK}

This section reviews literature related to the general domain of player experience analysis and provides some background on the facial expressions analysis techniques applied in ways similar to our goals.

\subsection{Player Experience Analysis}

We build on the novel perspective that computer vision techniques can provide to automatically infer gameplay experience metrics [27]. The motivations for this research is derived from the state of current research and practice in the area of player experience analysis, which is broadly categorized into qualitative and quantitative methods.

Qualitative methods involve the collection and analysis of subjective data for games and this often includes direct observations, interviews and think-aloud protocols. These methods are most common amongst game practitioners and usually require formal playtest sessions in artificial play environments [26]. Although these methods have been shown to usually reflect accurate states, they have several shortcomings. Firstly, they might inhibit true play experiences, as the players might not be totally at ease when someone is watching or questioning them. Players might not be able to properly self-articulate their play experiences concurrently during gameplay and might not even remember important details when post interviews are performed. Secondly, the sessions also often require a lot of time and resources to conduct and analyze. Hence there is a need for more efficient, accurate and versatile (ability to conduct in non-laboratory settings) ways to perform player experience analysis.

These reasons have driven much research towards quantitative methods that work on objective data. Quantitative methods have the potential to represent true player experiences in the game and are able to continuously capture a more diverse body of information. Common approaches include telemetry and psychophysiology.

Telemetry primarily deals with the logging of player in-game interactions to build player models, and several studies have been performed $[30,16,18,11]$. The advantage of Telemetry over qualitative methods is that it is non-disruptive and that it can continuously capture objective gameplay statistics in non-laboratory settings. However, the data is limited to the in-game actions available to the player and events in the game world. Hence these "virtual observations" do not capture full experiences and might not even represent the true experiences of the player in real life. For example, a player might take a long time to clear a level, but he might be having a high level of arousal in real life having fun exploring the level or simply stimulated by the aesthetics.

Psychophysiology is the other main branch of quantitative player experience research, which consists of methods to infer psychological states from physiological measurements, that commonly include electrodermal activity (EDA), electromyography (EMG), electrocardiogram (ECG), electroencephalography (EEG), body temperature and pupil dilations. Current work [13, 19, 21, 30, 8] mostly involve inferring emotional valence and arousal by employing a combination of the measurements. Amongst them, EDA and EMG seems to be most popular as they correspond accurately to emotional dimensions of arousal and valence respectively [23]. Similar to telemetry, physiological measurements are able to capture player experiences continuously in real-time. In addition, physiological data represent the real life experiences of the player. Unfortunately, most current approaches deal with expensive specialized equipment that are obtrusive, which are usually only viable in controlled laboratory settings.

These reasons have led to the position we have taken to propose the investigation of using a video-based approach to capture data in way that is more efficient, versatile, and does not affect natural gameplay. The approach we will start with is the established technique in computer vision - facial expressions analysis.

\subsection{Facial Expressions Analysis}

The first step in any facial expressions analysis system is to recognize facial expressions, and facial expression recognition is a fairly mature domain in computer vision with techniques that boast a high level of accuracy and robustness $[2,17,4,14]$. For example, Buenaposada et al. [4] have reported an $89 \%$ recognition accuracy in video sequences in unconstrained environments with strong changes in illumination and face locations.

In terms of using it for analysis of user experiences, there has been a limited number of works performed in non-game applications [3, 29]. Branco [3] showed some encouraging results evaluating positive and negative expressions of users of an online shopping website. Zaman and Shrimpton-Smith [29] evaluated an automated facial expressions analysis system to infer emotions that users had whilst performing common computer usage tasks. They generally reported a high 
level of correlation between the system's findings and human expert analyses. In other domains, general emotion detection based on facial expression recognition $[12,1]$ have also shown promising results.

\section{AUTOMATED PLAYER EXPERIENCE DE- TECTION FRAMEWORK}

The proposed eventual framework is a supervised learning system that maps facial expression features to gameplay experience metrics. An overview of our framework is as shown in Figure 1. There are basically two major system components, the Facial Expression Recognizer and the Play Classifier, which will be elaborated in Sections 3.1 and 3.2 respectively. Note however, that for this study, we are only evaluating the Facial Expression Recognizer. This overview of the eventual framework is to provide a holistic perspective of the project.

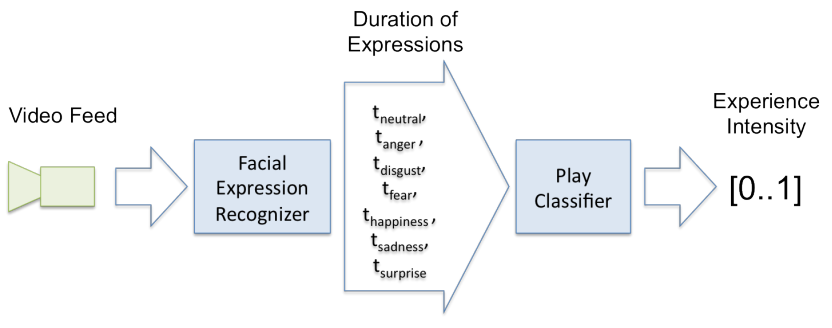

Figure 1: Overview of the automated player experience detector framework. The player's captured video is fed into the Facial Expression Recognizer which outputs the six basic emotions[9] and their durations. These features are then input into the Play Classifier which determines the intensity of a gameplay experience metric (like flow [28]).

\subsection{Facial Expression Recognizer}

Our implementation of the facial expression recognition is based on deformable model fitting. It is principled on the concept of learning independent image patches centered on landmarks on the face and has shown superior performance to holistic approaches (refer to [24] for a details of the technique). An important advantage of using this method is that it requires no training and no user intervention throughout the whole tracking, which is aligned with our goals of providing a non-intrusive method of data collection. This implementation represents the state-of-the-art in facial feature tracking which leads to the highest recognition rates in unconstrained video environments [6]. It should be noted that at this stage, improving the facial expression recognition is not our primary goal. Our primary goal is in investigating the feasibility of current state-of-the-art automated facial expression recognition in inferring play experiences.

For the duration of play for each player, the system will continuously detect the duration of each of the six basic expressions [9], namely anger, disgust, fear, happiness, sadness, surprise (with neutral as the baseline). The use of these six basic expressions, as opposed to the more detailed Facial Action Coding System (FACS) [10] is a conscious decision due to the fact that FACS action unit recognition being still an open problem $[15,14]$. In the future, we will gradually investigate the feasibility of using FACS as FACS recognition improves. Nevertheless, these six expressions has been shown to be a universal basis of emotions across diverse cultures. This approach also ensures that we have a comprehensible record of data for expert analyses of the data when needed, or as a complementary verification to the automatic analyses.

\subsection{Play Classifier}

The different emotions and their durations are fed into the play classifier in which the intensity of the experience metric (for example flow or immersion) will be determined as the output. A feed-forward Artificial Neural Network (ANN) is used here due to its success in prior work involving facial expressions [7].

After the game, the player will fill in a corresponding questionnaire (for example if the experience metric is flow, the Flow State Scale (FSS) [28] questionnaire will be used) so as to determine an aggregated experience intensity score of their game. This score will be used to train the classifier. As training convergence is reached, the system can then be used reliably to automatically infer experience intensities.

\section{EVALUATION}

\subsection{Method}

To have a detailed initial feasibility study, our approach has been primarily qualitative with quantitative data to support the analyses. A repeated-measures design was used to collect the data. All participants had their on-screen actions and facial video captured in two play conditions. The two conditions are basically the two different games played - the first game being Portal 2 by Valve ${ }^{3}$, and the second game Draw My Thing by OMGPOP ${ }^{4}$. Portal 2 is a story-based first-person puzzle shooter in which players shoot portals in order to solve spatial puzzles. In Portal 2, the participants played the single-player story mode from the start. Draw My Thing is a social word trivia game where players take turns to draw a picture using the mouse or track pad, in a time-constrained setting, and lets the other player guess the correct word. In Draw My Thing, the participants played with a single human opponent.

After the end of the experiments, the facial videos were then fed through the facial expression recognizer and graphs were generated for each player. These were then consolidated to produce the analyses as shown in Section 4.5.

\subsection{Setup}

The apparatus setup consists of a 2011 version 15-inch Intel Core i7 Apple notebook with 8GB RAM, a three-button mouse, and a Logitech C920 webcam ${ }^{5}$ capable to capturing full 1080p High Definition video. The on-screen actions were captured using the Screenflow software ${ }^{6}$. The notebook was placed in an office with common fluorescent lighting.

\footnotetext{
${ }^{3}$ http://www.thinkwithportals.com/

${ }^{4}$ http://www.omgpop.com/games/drawmything

${ }^{5}$ http://www.logitech.com/en-au/webcam-

communications/webcams/devices/hd-pro-webcam-c920

${ }^{6} \mathrm{http}: / /$ www.telestream.net/screen-flow/
} 
The facial expression recognizer described in Section 3.1 was built on the ofxFaceTracker add-on ${ }^{7}$ in the openFrameworks $\mathrm{C}++$ toolkit $^{8}$ using the OpenCV library ${ }^{9}$ for the computer vision functions. For our initial pilots, we used 3 common player expressions, namely joy, surprise, and anger, with an additional neutral expression as the baseline.

\subsection{Participants}

Participants were recruited via university mailing lists which includes university employees, undergraduates and alumni. 12 participants ( 4 females) took part in the study aged between 20 and $48($ mean $=34$, STD $=8)$.

The participants represented a wide mix of player types. 4 participants indicated that they play games for more than 5 hours per week, 8 participants less than 5 hours per week and 1 participant do not play games at all. 10 participants indicated they enjoy playing first-person shooters, 5 participants enjoy role-playing games, 5 participants enjoy strategy games, 3 participants enjoy simulations, 2 participants enjoy puzzle games, and 3 participants enjoy playing social word and trivia games. 6 participants indicated that they have played the Portal Series and 3 have played Drawing games by OMGPOP.

\subsection{Procedure}

After indicating their informed consent in the study, participants were asked to fill in a background questionnaire to determine player demographics (with the results as described in Section 4.3 above). They then proceed to play the two games, first Portal 2, followed by Draw My Thing. Both games were played for 15 minutes one after another in an enclosed room by themselves. The opponent in Draw My Thing played against the participant from a separate room over the Internet.

A short brief on the structure of the session was given to participants before starting the experiments. There were no tutorials or practice sessions prior to gameplay and they were left to figure out the games themselves. The participants were also told to play as they normally would, and not to think of this as an experiment. After each game, participants filled the Game Experience Questionnaire (GEQ) [20] but the data collected from the questionnaire are not used for the purpose of this paper.

After playing both games, participants were asked to describe whether the presence of the camera or other aspects of the experimental setup affected their play experience. No compensation were given to the participants at the end of the experiment.

\subsection{Results}

This initial analysis was meant to provide a primarily qualitative analysis of the feasibility of facial expressions. Hence the full graphs for each play session of Portal 2 and Draw My Thing for each player are as shown in Figures 2, 3, 4 and 5. The data are intentionally presented in a raw and detailed

\footnotetext{
${ }^{7}$ https://github.com/kylemcdonald/ofxFaceTracker

${ }^{8}$ http://www.openframeworks.cc/

${ }^{9}$ http://sourceforge.net/projects/opencvlibrary/
}

manner, without any smoothing, and without eliminating any "ugly" graphs.

An overall view of the graphs show that a good variety of facial expressions (other than neutral) were exhibited and the variances of expressions for each participant were generally rich. This implies that automatically recorded facial expression data can be used to meaningfully infer player experiences. Take for example participant 1 in Portal 2 (Figure 2 ), who starts off the game with primarily neutral expressions (green line) with spikes (red line) of joy being exhibited. This corresponds well to the introduction sequence of Portal 2 which involves a robot making hilarious conversations with the participant. A video inspection of the encoding process also reveals that the readings accurately reflect this. The other observation is that the participant's anger intensity (blue line) gradually increases over time. This also corresponds exactly to the participants self report at the end of the session where he/she mentioned that it was increasing frustrating when he/she could not figure out how to solve the spatial puzzle in the very first game room. Similar rich observations can be inferred from the graphs of the other players which implies that automatic analyses might be possible in future iterations of this research.

When visually inspecting the encoding process, we observed a number of participants placing their hands on their faces while playing Draw My Thing (which requires only one hand when drawing), or when watching cut scenes in Portal 2. Minor occlusions did not affect the readings but exaggerated hand placements resulted in empty readings during these instances. We have recorded all the empty readings as empty plots in the graph (the disjoint space between each line). Substantial empty readings can be seen in participants 4,6 and 9 whilst playing Draw My Thing (Figures 4 and 5).

To investigate the true feasibility of a video-based approach, we did not instruct the participants to consciously stay within the cameras vision, and have purposely included participants' charts with lots of empty readings. For example, one extreme case was participant 4 in Draw My Thing which had a large number of empty readings as seen in Figure 5. Upon a visual inspection of the video, participant 4 slouched very low when playing, with a section of his/her face outside of the camera. But this is not a common occurrence as can be verified from the graphs.

Summarized graphs of the averages across all the participants for Portal 2 and Draw My Thing are as shown in Figures 6 and 7 respectively. Here we can see that there is a larger amount of variance in the curves from Draw My Thing (Figure 7) than Portal 2 (Figure 6). This can again be verified from a visual inspection of the video that participants were more expressive in Draw My Thing than Portal 2. This indicates that the social element (of playing with another human) might elicit more facial expressions.

At the end of the entire session, when participants were asked to describe whether the presence of the camera or other aspects of the experimental setup affected their play experience, responses generally described that they were not conscious of the fact that their actions and their video was being recorded. For example some of the responses were: 


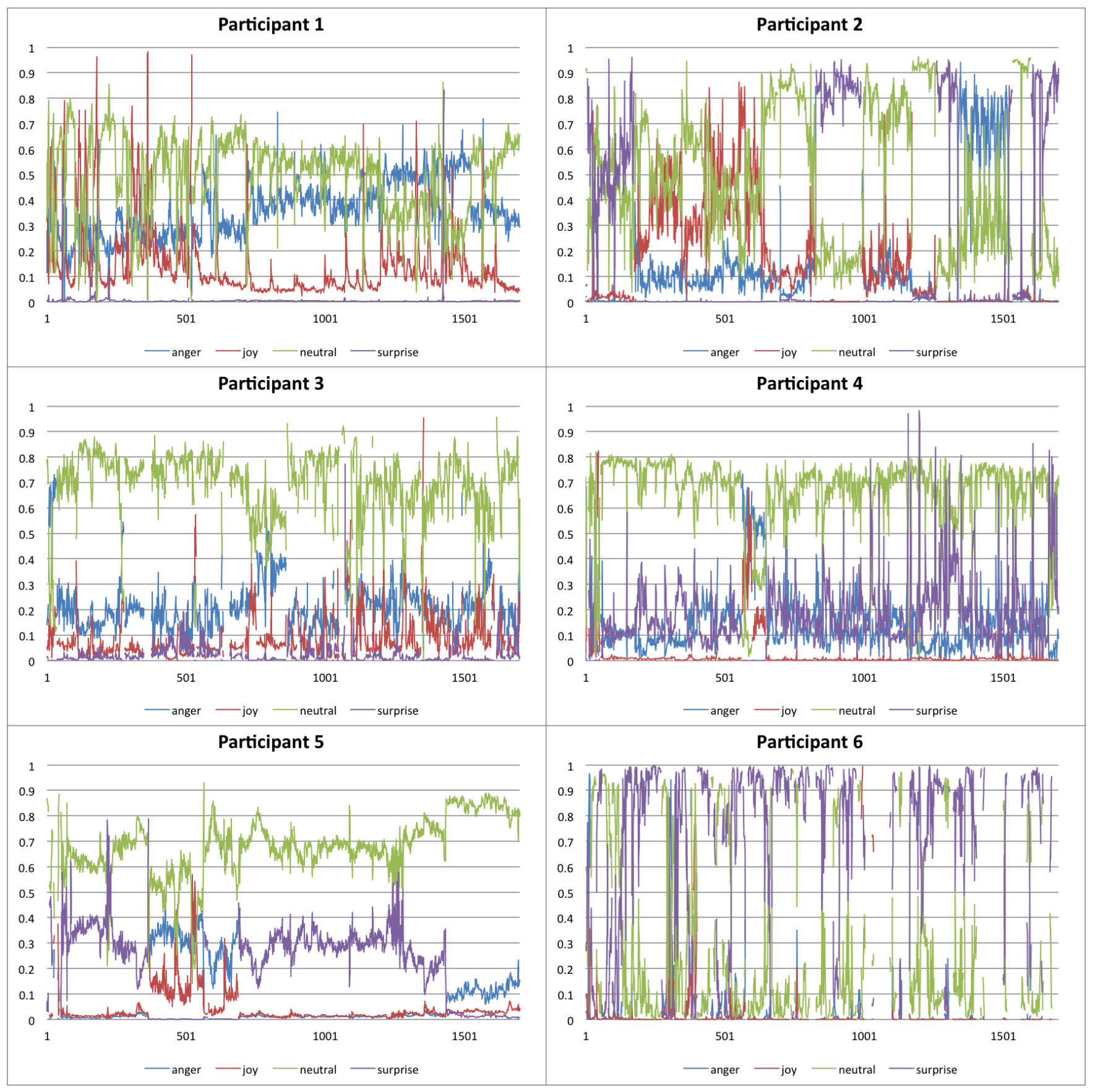

Figure 2: Probabilities over time for Portal 2 for participants 1 to 6: plot of expression probabilities (y-axis) against frame count (x-axis). Probabilities represent intensities of the respective expressions in that instance. 


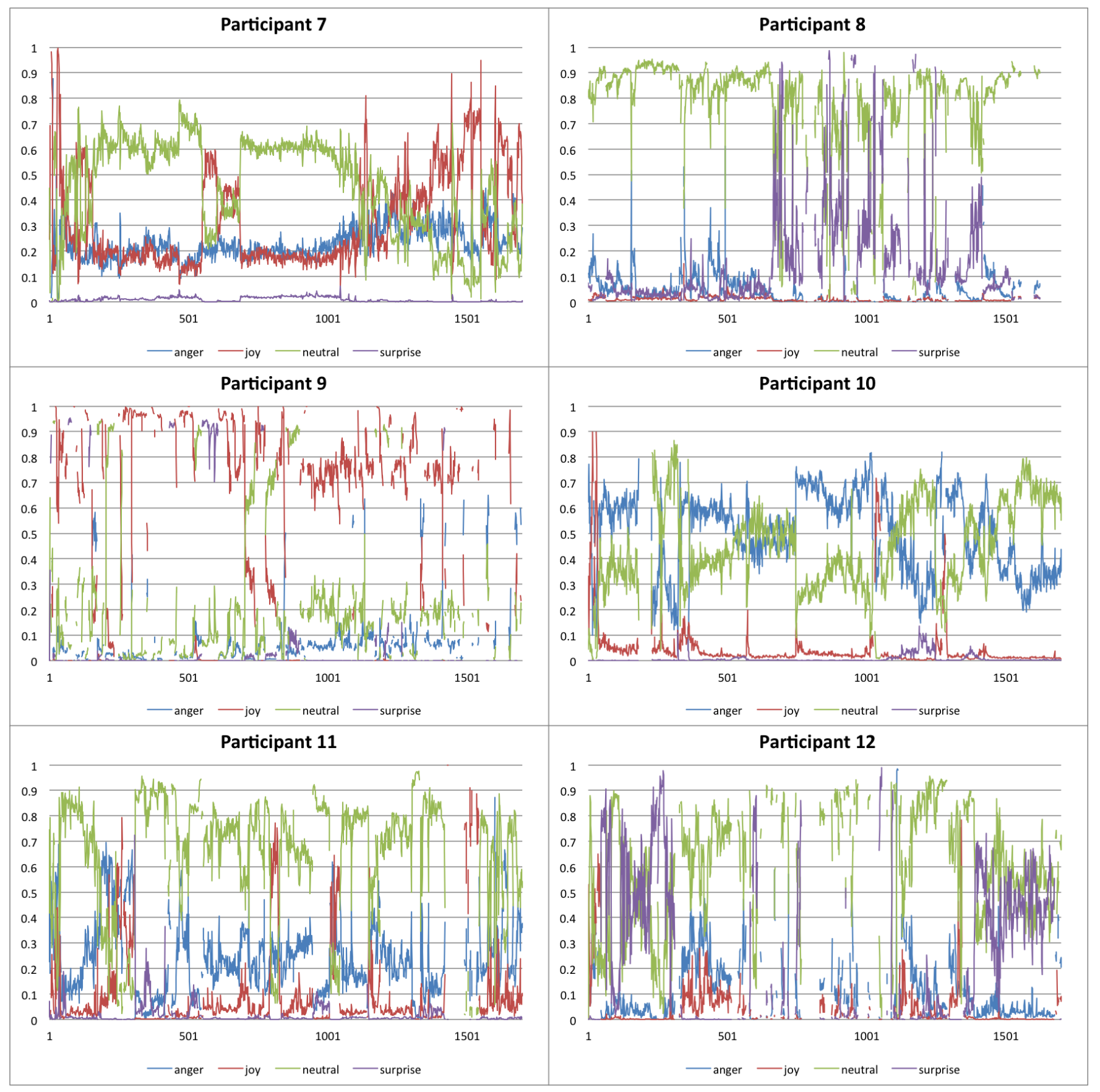

Figure 3: Probabilities over time for Portal 2 for participants 7 to 12: plot of expression probabilities (y-axis) against frame count (x-axis). Probabilities represent intensities of the respective expressions in that instance. 


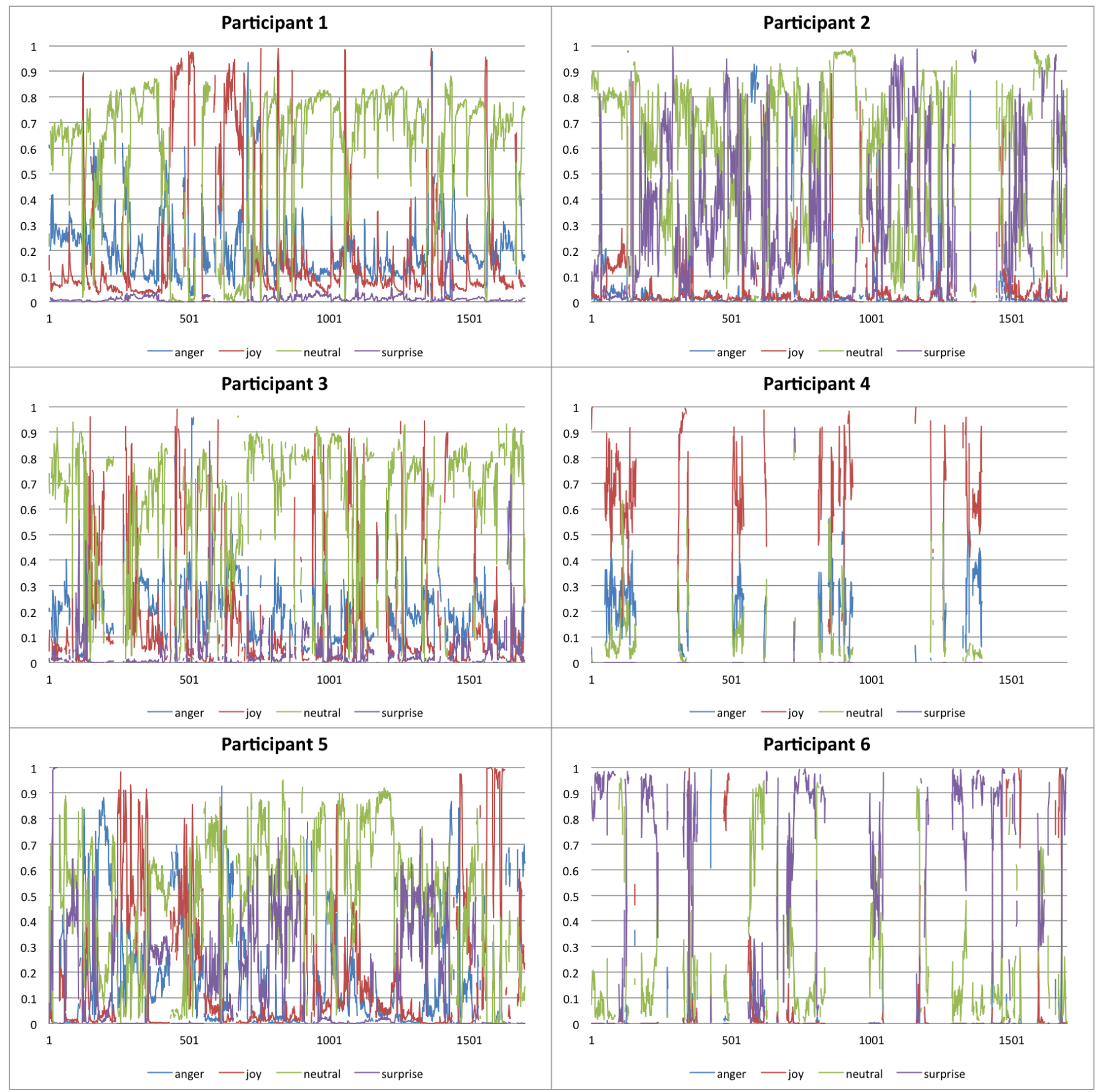

Figure 4: Probabilities over time for Draw My Thing for participants 1 to 6: plot of expression probabilities (y-axis) against frame count (x-axis). Probabilities represent intensities of the respective expressions in that instance. 


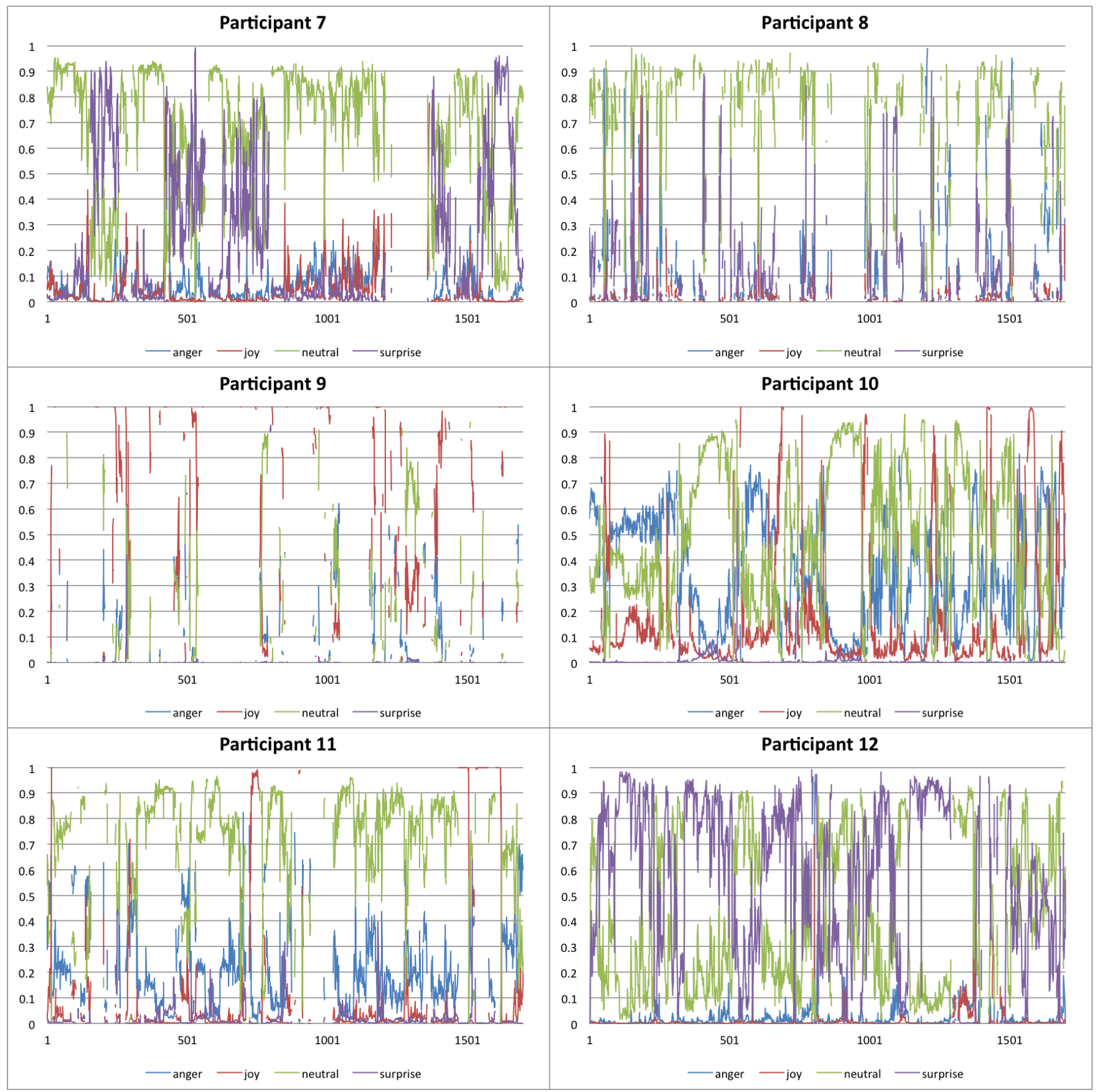

Figure 5: Probabilities over time for Draw My Thing for participants 1 to 6: plot of expression probabilities (y-axis) against frame count (x-axis). Probabilities represent intensities of the respective expressions in that instance. 
"i didn't think about being recorded...it was unobtrusive"

"Forgot all about the video recording!!!"

"Not really - only when I switched between games, or was waiting for a game."

However, some participants did appear to be slightly affected:

"the video recording wasn't affecting me too much.
However, I guess I would have shown more frus-
tration/anger if the video recording was not present."

"The knowledge that I am being recorded and that it is based upon my expressions, sometimes make me realize that I'm not just playing and I exit the state of mind that I am usually in when playing games."

These responses show promise that a video-based approach is indeed largely unobtrusive to gameplay. Perhaps alterations to the way the recording hardware was presented might improve this even further. A thorough study on obtrusiveness will be explored in future research.

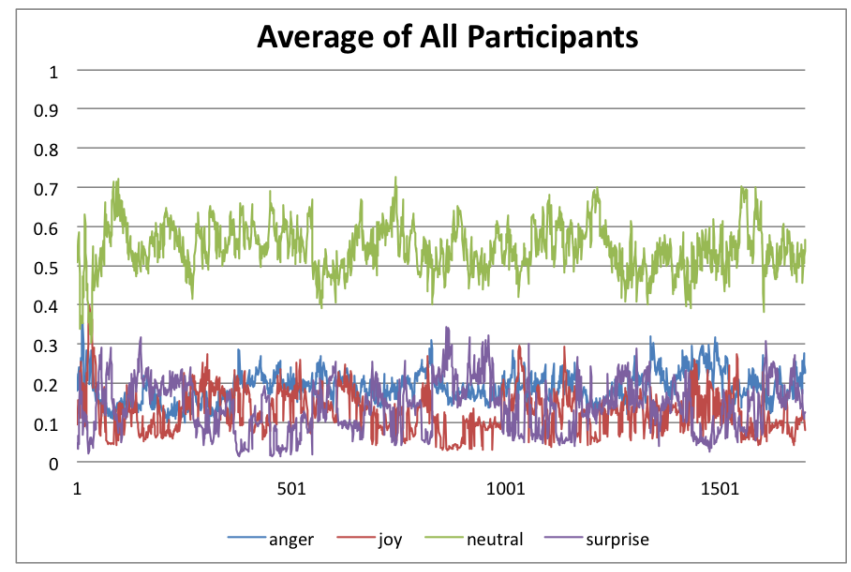

Figure 6: Average probabilities of all participants over time for Portal 2: plot of expression probabilities (y-axis) against frame count ( $\mathrm{x}$-axis). Probabilities represent intensities of the respective expressions in that instance.

\section{CONCLUSION}

We have performed an initial evaluation on using automated facial expressions analysis to infer player experiences. This approach builds on existing research aimed at devising a non-disruptive and non-obtrusive player experience analysis method that can be employed to automatically calculate player experience metrics.

A key finding is that each participants' graph represent a rich body of player experience data. This implies that there

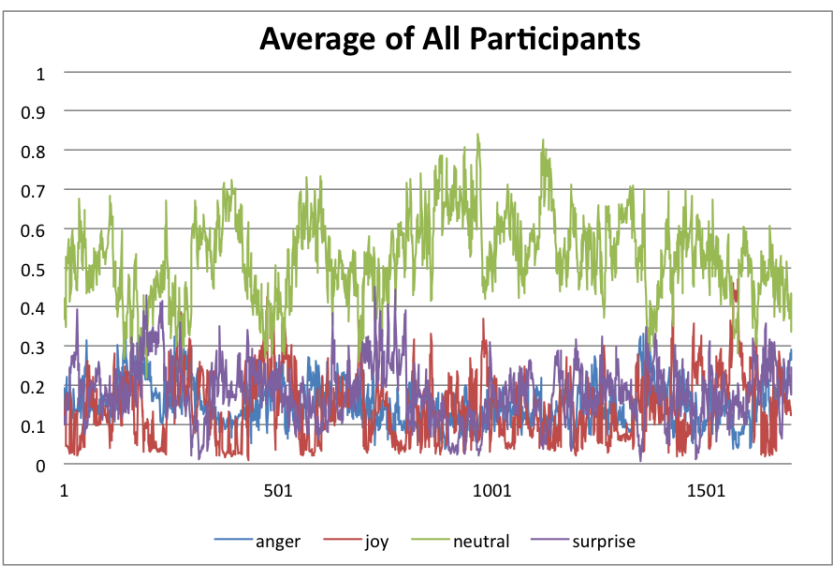

Figure 7: Average probabilities of all participants over time for Draw My Thing: plot of expression probabilities (y-axis) against frame count (x-axis). Probabilities represent intensities of the respective expressions in that instance.

are sufficient information from automatically captured facial expressions to infer player experiences. The results here can already enable a human to make meaningful analyses (as demonstrated in Section 4.5), but our ultimate goal is to be able to perform these analyses automatically using the play classifier. This will be the next major step in this research.

The other observation is that games with a social element to it (like Draw My Thing) induce more varied facial expressions than games without (like the single player campaign in Portal 2). This implies that the facial expression approach might be more suited to evaluate player experiences of this nature. Although this limits the scope of applicability, there is no lack of demand as networked multiplayer games represent a huge proportion of games on the market. Multiplayer options are also commonly seen in games a primary singleplayer focus (like Portal 2).

In terms of limitations, missed readings were abundant in some graphs due to occlusions. This would be rather inevitable in natural gameplay for players with a lot of movement around the face. One way would be to investigate webcams with a larger field of view, and the other way is to investigate computer vision methods that can handle occlusions better. As our aim is not to advance the state-ofthe-art in facial expression recognition, we plan to evaluate several other recent recognition algorithms as well as commercial solutions if they serve the purpose better.

In conclusion, the results in this paper indicates a promising starting point for an eventual framework that can easily integrate into existing games to provide feedback even after they have been launched. Using facial expressions, we can capture a rich and continuous representation of reallife player responses in authentic play situations, enabling a finer-grained analysis of gameplay experiences. Using an automated computer analysis method, it also resolves the problem of privacy concerns by playtesters who are not comfortable having their video viewed by others. It will have importance applications for game companies who need to 
easily analyze real life user experience data.

\section{REFERENCES}

[1] T. Baltrusaitis, D. McDuff, N. Banda, M. Mahmoud, R. E. Kaliouby, P. Robinson, and R. Picard. Real-time inference of mental states from facial expressions and upper body gestures. Face and Gesture 2011, pages 909-914, Mar. 2011.

[2] M. S. Bartlett, J. C. Hager, P. Ekman, and T. J. Sejnowski. Measuring facial expressions by computer image analysis. Psychophysiology, 36(2):253-63, Mar. 1999.

[3] P. Branco. Computer-based facial expression analysis for assessing user experience. PhD thesis, 2006.

[4] J. M. Buenaposada, E. Muñoz, and L. Baumela. Recognising facial expressions in video sequences. Pattern Analysis and Applications, 11(1):101-116, Oct. 2007.

[5] A. Chapin. THE FUTURE IS A VIDEOGAME. Canadian Business, 84(4):46-48, 2011.

[6] J. F. Cohn and M. A. Sayette. Automated Facial Coding. Behavioral Research Methods, pages 1-33, 2010.

[7] M. N. Dailey, G. W. Cottrell, C. Padgett, and R. Adolphs. EMPATH: a neural network that categorizes facial expressions. Journal of cognitive neuroscience, 14(8):1158-73, Nov. 2002.

[8] A. Drachen, L. Nacke, G. Yannakakis, and A. Pedersen. Correlation between heart rate, electrodermal activity and player experience in First-Person Shooter games. In Proceedings of the 5th ACM SIGGRAPH Symposium on Video Games, pages 49-54. ACM, 2010.

[9] P. Ekman and W. V. Friesen. The repertoire of nonverbal behavior: Categories, origins, usage, and coding. Semiotica, 1(1):49-98, 1969.

[10] P. Ekman, W. V. Friesen, and J. C. Hager. Facial Action Coding System, volume 48. A Human Face, 2002.

[11] A. Gagne, M. Seif El-Nasr, and C. Shaw. A Deeper Look at the use of Telemetry for Analysis of Player Behavior in RTS Games. In International Conference on Entertainment Computing ICEC, 2011.

[12] M. Ghijsen. Facial Expression Analysis for Human Computer Interaction. Affective Computing, IEEE Transactions on, 2(3):147-161, 2004.

[13] R. L. Mandryk, M. S. Atkins, and K. M. Inkpen. A continuous and objective evaluation of emotional experience with interactive play environments. Proceedings of the SIGCHI conference on Human Factors in computing systems - CHI '06, page 1027, 2006.

[14] D. McDuff, R. el Kaliouby, K. Kassam, and R. Picard. Acume: A new visualization tool for understanding facial expression and gesture data. In Face and Gesture 2011, pages 591-596. IEEE, Mar. 2011.

[15] D. McDuff, R. E. Kaliouby, and K. Kassam. Affect valence inference from facial action unit spectrograms. Computer Society Conference on Computer Vision and Pattern Recognition Workshops, 2010.

[16] B. Medler, M. John, and J. Lane. Data cracker: developing a visual game analytic tool for analyzing online gameplay. In Proceedings of the 2011 annual conference on Human factors in computing systems, pages 2365-2374. ACM, 2011.

[17] P. Michel and R. El Kaliouby. Real time facial expression recognition in video using support vector machines. Proceedings of the 5th international conference on Multimodal interfaces - ICMI '03, page 258, 2003.

[18] D. Moura, M. Seif El-Nasr, and C. D. Shaw. Visualizing and Understanding Players âÁŹ Behavior in Video Games : Discovering Patterns and Supporting Aggregation and Comparison. In SIGGRAPH, pages 2-7, 2011.

[19] L. Nacke and C. Lindley. Flow and immersion in first-person shooters: measuring the player\&\#39;s gameplay experience. Proceedings of the 2008 Conference on Future, pages 81-88, 2008.

[20] L. E. Nacke. Affective Ludology: Scientific Measurement of User Experience in Interactive Entertainment. PhD thesis, 2009.

[21] L. E. Nacke, M. N. Grimshaw, and C. a. Lindley. More than a feeling: Measurement of sonic user experience and psychophysiology in a first-person shooter game. Interacting with Computers, 22(5):336-343, Sept. 2010.

[22] B. Reeves, J. Cummings, and J. Scarboroug. Government Uses for Games and Virtual Worlds : Optimizing Choices for Citizens and Government Workers in the Areas of Energy Efficiency, Educational Assessment, Worker Productivity, Safety and Health, and Quality. Technical report, 2010.

[23] J. A. Russell. A circumplex model of affect. Journal of Personality and Social Psychology, 39(6):1161-1178, 1980.

[24] J. M. Saragih, S. Lucey, and J. F. Cohn. Deformable Model Fitting by Regularized Landmark Mean-Shift. International Journal of Computer Vision, 91(2):200-215, Sept. 2010.

[25] P. Sweetser and P. Wyeth. GameFlow: a model for evaluating player enjoyment in games. Computers in Entertainment (CIE), 3(3):3-3, 2005.

[26] C. Tan and A. Johnston. Towards a Nondisruptive, Practical, and Objective Automated Playtesting Process. In Workshops at the Seventh Artificial Intelligence and Interactive Digital Entertainment Conference, pages 25-28, 2011.

[27] C. T. Tan and Y. Pisan. Towards Automated Player Experience Detection With Computer Vision Techniques. In CHI Workshop on Game User Experience, 2012.

[28] G. Tenenbaum, G. J. Fogarty, and S. A. Jackson. The flow experience: a Rasch analysis of Jackson's Flow State Scale. Journal Of Outcome Measurement, 3(3):278-294, 1999.

[29] B. Zaman and T. Shrimpton-Smith. The FaceReader: Measuring instant fun of use. In Proceedings of the 4 th Nordic conference on Human-computer interaction: changing roles, number October, pages 457-460. ACM, 2006.

[30] V. Zammitto, M. Seif El-Nasr, and P. Newton. Exploring Quantitative Methods for Evaluating Sports Games. Signals, pages 8-11, 2010. 